\title{
miR-544a promotes the invasion of lung cancer cells by targeting cadherina I in vitro
}

This article was published in the following Dove Press journal:

OncoTargets and Therapy

4 June 2014

Number of times this article has been viewed

Xiaomei Mol,2,*

Fenghua Zhang 2,* $^{2}$

Hui Liang ${ }^{2}$

Ming Liu'

Huahui $\mathrm{Li}^{3}$

Haiping $\mathrm{Xia}^{3}$

'Key Laboratory of Marine Drugs, Ministry of Education, School of Medicine and Pharmacy, Ocean University of China, ${ }^{2}$ Qingdao Women and Children Hospital, ${ }^{3}$ Department of Laboratory Medicine, Qingdao Municipal Hospital, Qingdao, People's Republic of China

*These authors contributed equally to this work
Correspondence: Huahui Li

Qingdao Municipal Hospital, 5 Donghai

Zhong Road, Qingdao, Shandong

Province, People's Republic of China

Tel +8653288905172

Email Ihh39153@I63.com
Objective: To find out the effect of miR-544a on the invasion of lung cancer cells and to explore the underlying molecular mechanisms.

Methods: Micro-ribonucleic acid (miRNA) expression in two different invasive lung cancer cell lines 95C (low invasive ability) and 95D (high invasive ability) was analyzed by miRNA microarray and real-time quantitative polymerase chain reaction (PCR); miR-544a mimic was transfected to $95 \mathrm{C}$, and its invasion ability was detected by transwell migration assay; we predicted the candidate miRNA target genes by TargetScan (Whitehead Institute for Biomedical Research, Cambridge, MA, USA) software and verified the target genes by Western blot.

Results: The expression of miR-544a was significantly increased in 95D in miRNA microarray and quantitative PCR tests $(P<0.05)$. After being transfected with miR-544a mimic, the invasion ability of $95 \mathrm{C}$ was enhanced $(P<0.01)$. Moreover, transfection with miR-544a inhibitor decreased the invasion ability of 95D $(P<0.01)$. miR-544a possibly combined with CDH1 (E-cadherin) predicted by the TargetScan analysis. 95C with miR-544a mimic reduced the expression of CDH1 and improved the expression of vimentin, while 95D with miR-544a inhibitor improved the expression of $\mathrm{CDH} 1$ and reduced the expression of vimentin.

Conclusion: miR-544a can promote the invasion of non-small cell lung cancer by downregulation of $\mathrm{CDH} 1$ and upregulation of vimentin.

Keywords: NSCLC, non-small cell lung cancer, E-cadherin, microRNA, EMT

\section{Introduction}

Non-small cell lung cancer (NSCLC) occupies $80 \%$ of all types of lung cancer, ${ }^{1}$ and many cancer patients die of cancer invasion or metastasis. Therefore, it is necessary to do further study on the molecule markers that play an important role in the invasion or metastasis in NSCLC.

Mature micro-ribonucleic acids (miRNAs) consist of 22 nucleotides, and as negative regulators of gene expression, mainly recognize the complementary sequences in the $3^{\prime}$ untranslated regions (UTRs) of their target messenger RNAs. ${ }^{2}$ Many studies have revealed that miRNA not only participates in biological processes such as cell cycle, aging, and death but also is a hallmark of several pathological conditions, including cancer, exerting a causal role, as oncogenes or tumor suppressors. For example, miR-544a levels are reduced in glioma ${ }^{3}$ and invasive ductal carcinoma. ${ }^{4}$ miR-544a can be seen as the molecular marker of glioma and tumor suppressor. miR-544a exerts its carcinogenic factor, promoting cell cycle and cell proliferation of stomach cancer, by reducing $I R X 1$, one tumor suppressor gene. ${ }^{5}$ However, there has been little study on the role of miR-544a in NSCLC metastasis and invasion. Invasion and metastasis usually 
occur even after complete lung cancer resection; therefore, two different invasive lung cancer cell lines (95C and 95D) were used to explore the miR-544a levels, and predict the target gene and its potential downstream proteins. This study will provide insights into the role of miR-544a in lung cancer invasion and metastasis and the possible molecular mechanisms.

\section{Materials and methods Cell lines and reagents}

The 95D and 95C cells were purchased from the American Type Culture Collection and cultured in Dulbecco's Modified Eagle's Medium with 10\% fetal bovine serum (FBS). The 95D cells showed higher invasion, while the 95C cells showed lower invasion. Moloney murine leukemia virus reverse transcriptase (MMLV-RT) was purchased from Toyobo Corporation (Osaka, Japan).

\section{miRNA array}

$10^{6}-10^{7} 95 \mathrm{D}$ or $95 \mathrm{C}$ cells in logarithmic phase, conventional cell culture, and total RNA were extracted with TRIzol ${ }^{\circledR}$ (Life Technologies, Carlsbad, CA, USA). miRNA array was performed in Beijing Bo Ao Capitalbio Corporation with $40 \mu \mathrm{g}$ total RNA. Bo Ao miRNA array included 469 probes. U6 and transfer RNA were used as inner control, and eight synthetic RNAs (20-30 nucleotides) were used as exogenous control. Hex was used as positive control, and 50\% DMSO (dimethyl sulfoxide) as negative control (NC). Loess normalization was used to normalize the miRNA array data. Every RNA sample was performed in duplicate.

\section{Bioinformatics analysis}

The target gene was predicted by TargetScan (Whitehead Institute for Biomedical Research, Cambridge, MA, USA) software.

\section{Luciferase assays}

LightSwitch $^{\text {TM }}$ (Promega, Fitchburg, WI, USA) luciferase assay reagents are from Promega Corporation (Fitchburg, WI, USA). miRNA NC and miR-544a mimic combine with E-cadherin (CDH1) 3'UTR or CDH1 mutated 3' UTR and are transfected to $293 \mathrm{~T}$ cells for 24 hours, respectively. Assay reagents were added to each well according to the manufacturer's instructions (Promega Corporation). Firefly luciferase (FLuc) and renilla luciferase (RLuc) were counted by a chemiluminescence meter (Promega Corporation). Luciferase expression was recorded as relative light units (RLuc/FLuc) to estimate whether $C D H 1$ is the target gene of miR-544a.

\section{Quantitative polymerase chain reaction (PCR)}

Total RNA was extracted from 95C or 95D with TRIzol and reverted to complementary deoxyribonucleic acid (cDNA) by MMLV-RT. Quantitative PCR was performed using a PCR Detection System (Bio-Rad Laboratories, Hercules, CA, USA) with the use of SYBR ${ }^{\circledR}$ Green I Premix Ex Taq $^{\text {TM }}$ (Takara Bio Inc, Shiga, Japan). Specific primers for miR-544a were designed by Rui Bo Company (Guangzhou, People's Republic of China). Reagents include $2 \times$ Mix SYBR Green I $10 \mu \mathrm{L}, 10$ pM primers $0.25 \mu \mathrm{L}$, cDNA $1 \mu \mathrm{L}$, and double-distilled $\mathrm{H}_{2} \mathrm{O}$ up to $20 \mu \mathrm{L}$. The reaction protocol included an initial step of 120 seconds at $95^{\circ} \mathrm{C}$. Each PCR cycle involved denaturation $\left(95^{\circ} \mathrm{C}, 30\right.$ seconds), annealing $\left(60^{\circ} \mathrm{C}, 35\right.$ seconds), and extension $\left(72^{\circ} \mathrm{C}, 20\right.$ seconds $)$ for 40 cycles. Fluorescence was measured at each cycle. The relative fold change of miR-544a expression was quantified as $2^{-\Delta \Delta \mathrm{Ct}}$, where $\Delta \Delta \mathrm{Ct}$ was $\mathrm{Ct}$ (target genes) - Ct (housekeeping genes). We selected small nuclear RNA U6 as housekeeping gene. The nucleotide sequence of the upstream primer is TGGCACCCAGCACAATGAA; and the downstream primer, CTAAGTCATAGTCCGCCTAGAAGCA.

\section{Transfection}

95C was transfected with miR-544a mimic and $\mathrm{NC}$, respectively, while $95 \mathrm{D}$ was transfected with miR-544a inhibitor and $\mathrm{NC}$, according to the manufacturer instructions for Lipofectamine 2000 (Life Technologies, Carlsbad, CA, USA): $1 \times 10^{5} 95 \mathrm{C}$ or $95 \mathrm{D}, 50 \mu \mathrm{L} 1 \times$ buffer, $2.5 \mu \mathrm{L}$ miR-544a mimic, miR-544a inhibitor, or NC. After being transfected for 36 hours, the cells were applied to transwell migration assay and Western blot.

\section{Transwell migration assay}

Put Matrigel ${ }^{\mathrm{TM}}$ (BD Biosciences, San Jose, CA, USA) at $4^{\circ} \mathrm{C}$ overnight. Dilute Matrigel with serum-free Roswell Park Memorial Institute (RPMI)-1640 at a 1:9 dilution. Add 50 $\mu \mathrm{L}$ diluted Matrigel to each transwell hole and put it at $37^{\circ} \mathrm{C}$ for $3 \sim 4$ hours. A total of $5 \times 10^{4}$ transfected cells (95C transfected with miR-544a mimic and NC; 95D with miR-544a inhibitor and NC) suspended in $200 \mu \mathrm{L}$ serum-free RPMI-1640 medium was placed into the upper chamber. Outside the upper chamber, $500 \mu \mathrm{L}$ 10\% FBS-RPMI-1640 medium was added. After 24 hours of incubation, cells were washed with PBS three times, and cells remaining on the upper membrane were carefully removed. Cells that had migrated through the membrane were fixed with methanol and stained with hematein for 5 minutes. Finally, the migrated cells were imaged and counted 
at $10 \times$ magnification using a Leica DC 300F microscope (Olympus Corporation, Tokyo, Japan).

\section{Western blot}

95C-NC, 95C-miR-544a-mimic, 95D-miR-544a-inhibitor, and $95 \mathrm{D}-\mathrm{NC}$ were lysed by $100 \mu \mathrm{L}$ radioimmunoprecipitation assay buffer (RIPA; Sigma-Aldrich, St Louis, MO, USA). The protein concentration was detected by bicinchoninic acid (BCA) protein assay kit (HyClone-Pierce, Logan, UT, USA). Protein $(20 \mu \mathrm{g})$ was loaded on 12\% SDS-PAGE (sodium dodecyl sulfate polyacrylamide gel electrophoresis) ( $120 \mathrm{~V}, 1.5$ hours), transferred to PVDF (polyvinylidene difluoride) membrane and blotted by $5 \%$ skim milk powder for 2 hours. Membranes were probed with primary antibodies $(1: 10,000)$ of CDH1 (Abcam, Cambridge, MA, USA), vimentin (Santa Cruz Biotechnology, Inc., Dallas, TX, USA), $\alpha$-tubulin (Santa Cruz Biotechnology, Inc.), and horseradish peroxidase (HRP)-conjugated secondary antibody (Santa Cruz Biotechnology, Inc.). Protein expression was quantitatively assessed using a HRP-ECL scanner (Lenovo, Beijing, People's Republic of China).

\section{Statistical analysis}

Student-Newman-Keuls-q (SNK-q) is used to compare pairs of means of multiple data, and Student's $t$-test is used for the difference in only two means. One-way ANOVA (analysis of variance) with SNK-q test for multiple comparisons was used to analyze the data from the transwell migration assay and Western blot using SPSS 15.0 (SPSS Inc., Chicago,

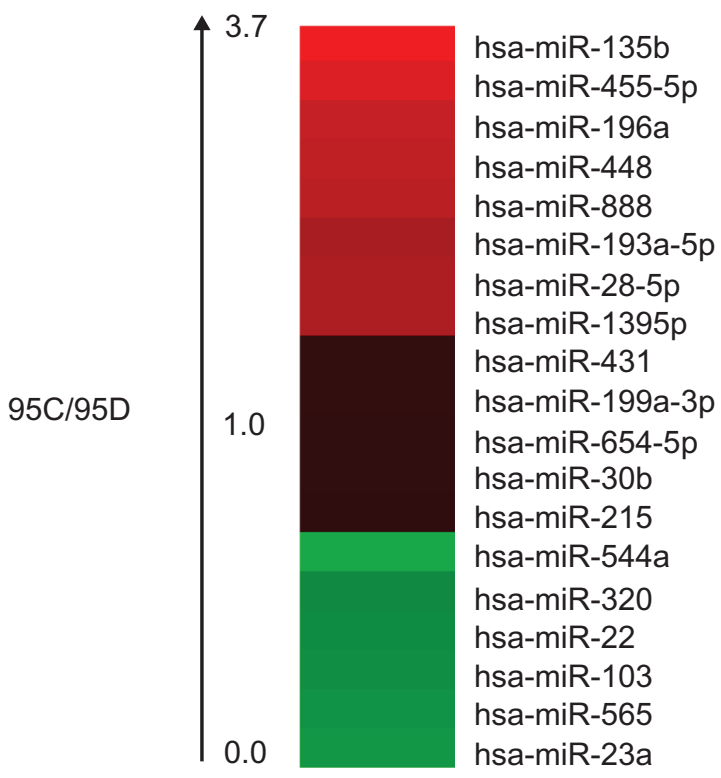

Figure I The ratio of miRNA levels in $95 \mathrm{C}$ and $95 \mathrm{D}$ by miRNA array. The ratio of miR-544a levels in 95C to 95D is below 1.0.

Abbreviations: miRNA, micro-ribonucleic acid; hsa, homo sapiens; 95C, low invasive ability lung cancer cell line; 95D, high invasive ability lung cancer cell line.

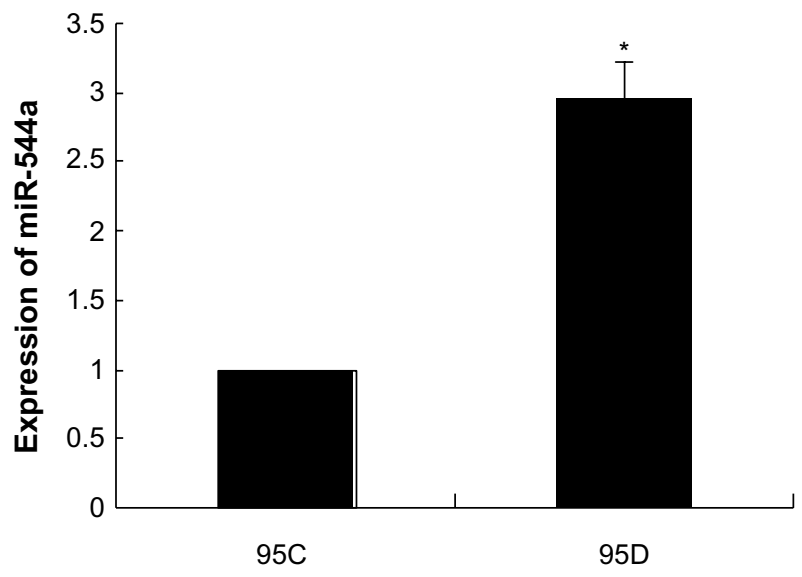

Figure 2 miR-544a levels in $95 \mathrm{C}$ and 95D by quantitative polymerase chain reaction. The relative level of miR-544a in 95D was almost three times that in $95 \mathrm{C}$ cells. Note: $* P<0.05$.

IL, USA) software. Student's $t$-test was used to analyze the results of quantitative PCR. Data are shown as mean \pm standard deviation. Differences of $P<0.05$ were considered statistically significant.

\section{Results}

\section{C and 95D expressed different levels of miR-544a}

In the miRNA array, we analyzed the miR-544a levels in 95C and $95 \mathrm{D}$ cells, and the results showed that the ratio of miR-544a levels in 95C and 95D was very low, indicating that the level of miR-544a in 95D was higher than that in 95C (Figure 1). To verify the results of the miRNA array, quantitative PCR was carried out, and the results showed that the relative level of miR544a in 95D was almost threefold that in 95C cells (Figure 2 and Table 1). All of these results showed that there is a higher level of miR-544a in 95D than in 95C cells.

\section{$\mathrm{CDHI}$ is one of the target genes of miR-544a}

TargetScan was used to predict the target gene of miR-544a, and the results showed that miR-544a possibly combined with

Table I miR-544a levels in $95 \mathrm{C}$ and 95D by quantitative polymerase chain reaction $(n=9)$

\begin{tabular}{ll}
\hline Group & $\mathbf{2}^{-\Delta \Delta C t}($ mean \pm SD $)$ \\
\hline $95 C$ & $1.00 \pm 0.00$ \\
$95 D$ & $2.95 \pm 0.26$ \\
$t$ & 12.94 \\
$P$ & $<0.05$ \\
\hline
\end{tabular}

Abbreviations: SD, standard deviation; $\mathrm{Ct}$, cycle of threshold; $\Delta \Delta \mathrm{Ct}, \mathrm{Ct}$ (miR544a-small nuclear RNA U6); 95C, low invasive ability lung cancer cell line; 95D, high invasive ability lung cancer cell line. 


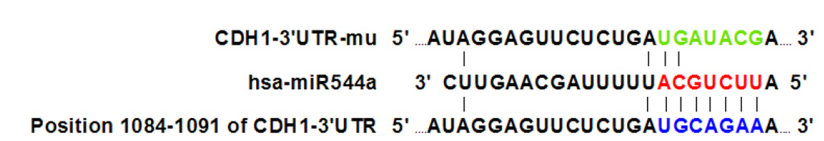

Figure 3 miR-544a can combine with 3'UTR of CDHI.

Notes: Green sequence: mutate sequence of $C D H I$. Red sequence: seed sequence of miR544a. Blue sequence: mutate sequence of $C D H I$.

Abbreviations: UTR, untranslated region; CDHI, E-cadherin ; hsa, homo sapiens; mu, mutate.

CDH1 (Figure 3). CDH1 is one of the cell adhesion molecules on cell membranes, maintaining cell integrity and exerting the ability of cell contact inhibition. To verify the interaction between $C D H 1$ and miR544a, luciferase assay was carried out. As shown in Figure 4 and Table 2, miR-544a mimic could combine with CDH1 3'UTR and inhibit the expression of the reporter gene $(0.63 \pm 0.13)$. When CDH1 3'UTR mutated, miR544a mimic could not combine with CDH1 mutated 3'UTR and the expression of the reporter gene $(1.31 \pm 0.04)$ increased $(q=10.12, P<0.01)$. These results suggest that $\mathrm{CDH} 1$ is one of the target genes of miR-544a.

\section{miR-544a enhances the migration of NSCLC cells}

To study the effect of miR-544a on cancer cell migration, a transwell migration assay was performed, and the results are shown in Figure 5. After transfection with miR-544a mimic, migrated cells numbered more than that of 95C-NC $(q=18.67$, $P<0.01)$. When cells were treated with 95D-miR-544a inhibitor, migrated cells numbered less than that of 95D-NC $(q=18.67, P<0.01)$ (Table 3). These results suggest that miR-544a could enhance the migration of NSCLC cells.

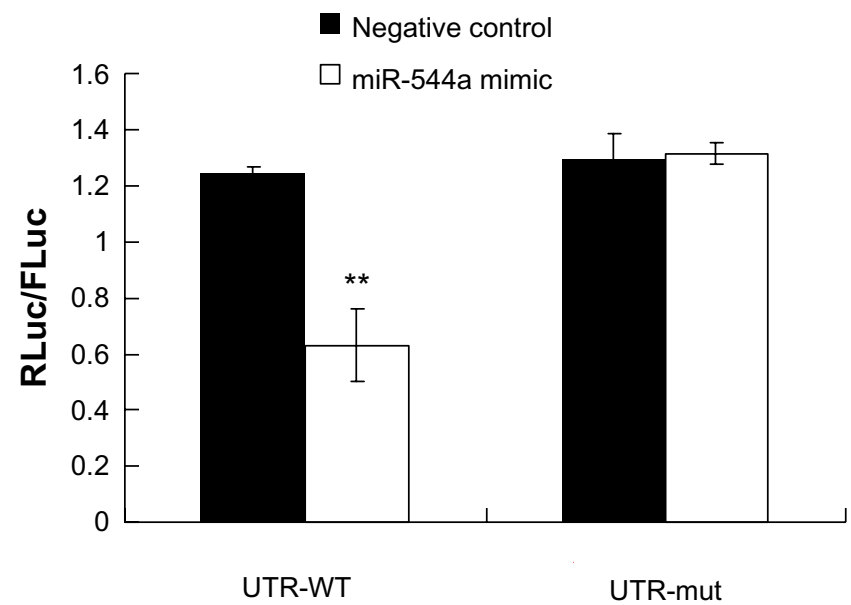

Figure 4 Verification of miR-544a target gene (CDHI) by luciferase assay. RLuc/ FLuc-ratio in cells with miR544a-mimic-CDHI $3^{\prime} U T R(0.63 \pm 0.13)$ was lower than that of miR-544a mimic-CDHI mutated $3^{\prime} U T R(I .3 I \pm 0.04, * * P<0.01)$. These results suggest that $\mathrm{CDHI}$ is one of the target genes of miR-544a.

Abbreviations: $\mathrm{CDHI}, \mathrm{E}-\mathrm{cadherin}$; FLuc, firefly luciferase; RLuc, renilla luciferase; UTR, untranslated region; mut, mutant; WT, wild type.
Table 2 Verification of miR-544a target gene (CDHI) by luciferase assay (mean $\pm S D ; n=9$ )

\begin{tabular}{ll}
\hline Group & RLuc/FLuc \\
\hline miR-544a mimic + CDHI 3'UTR & $0.63 \pm 0.13^{\mathrm{a}}$ \\
miR-544a mimic + CDHI mutated 3'UTR & $\mathrm{I} .31 \pm 0.04$ \\
miR-544a-NC + CDHI 3'UTR & $1.24 \pm 0.04$ \\
miR-544a-NC + CDHI mutated 3'UTR & $1.30 \pm 0.09$ \\
\hline
\end{tabular}

Notes: ${ }^{a}$ Compared with miR-544a mimic $+\mathrm{CDHI}$ mutated $3^{\prime} \mathrm{UTR}, \mathrm{q}=10.12$, $F=47.60, P<0.01$.

Abbreviations: $\mathrm{CDHI}$, E-cadherin; FLuc, firefly luciferase; RLuc, renilla luciferase; $\mathrm{SD}$, standard deviation; UTR, untranslated region.

\section{miR-544a downregulates CDHI}

To investigate whether miR-544a could affect the expression of CDH1, Western blot was performed. In cells treated with 95C-miR-544a mimic, the level of CDH1 decreased and that of vimentin increased compared with 95C-NC $(P<0.01)$. When treated with 95D-miR-544a inhibitor, the level of

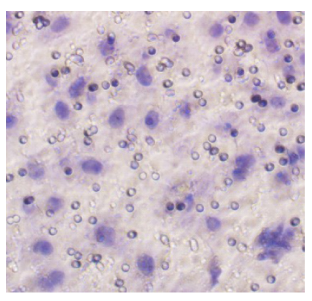

95C-miR-544a mimic

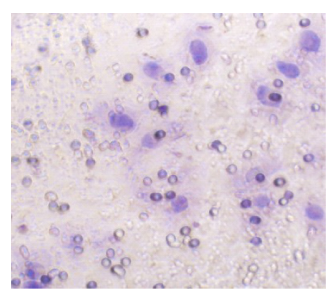

95D-miR-544a inhibitor

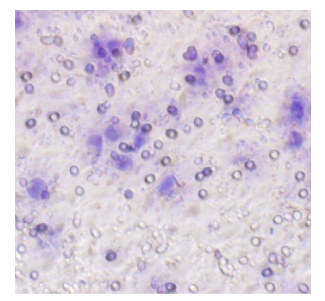

95C-NC

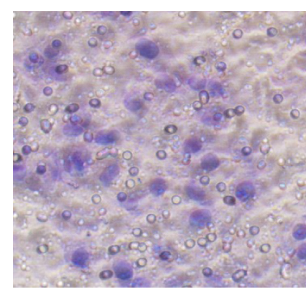

95D-NC

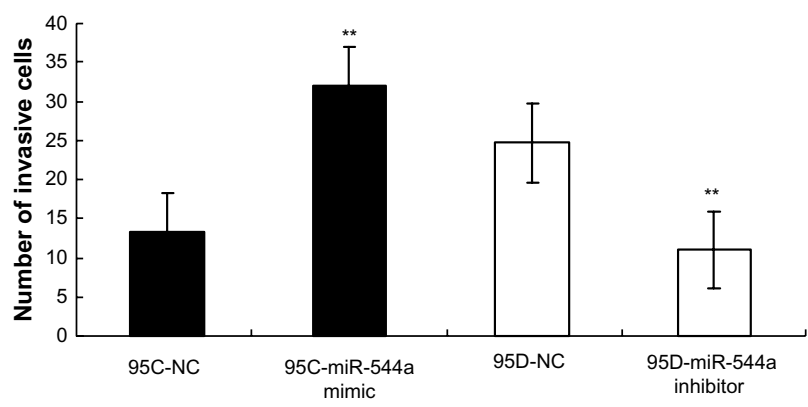

Figure 5 The effect of miR-544a on 95C and 95D migration by transwell migration assay $(\times 200)$. Migration cells in $95 \mathrm{C}-\mathrm{miR}-544$ a mimic were higher than that in $95 \mathrm{C}-\mathrm{NC}(32.00 \pm 1.00$ versus 13.33 $\pm 0.0058, * * P<0.01)$. Migration cells in 95D-miR544 a inhibitor were lower than that in 95D-NC $(11.00 \pm 1.00$ versus $24.67 \pm 0.58$, $* * P<0.0$ I). These results indicate that miR-544a can promote cell migration. Abbreviation: NC, negative control. 
Table 3 The effect of miR-544a on 95C and 95D migration by transwell migration assay

\begin{tabular}{ll}
\hline Group & Cells (mean \pm SD) $(\mathbf{n}=\mathbf{9})$ \\
\hline 95C-NC & $13.33 \pm 0.0058$ \\
95C-miR-544a mimic & $32.00 \pm 1.00^{\mathrm{a}}$ \\
95D-NC & $24.67 \pm 0.58$ \\
95D-miR-544a inhibitor & $11.00 \pm 1.00^{\mathrm{b}}$ \\
\hline
\end{tabular}

Notes: ${ }^{a}$ Compared with 95C-NC, $q=18.67, P<0.01$; ${ }^{b}$ compared with 95D NC, $q=13.67, P<0.01, F=436.46$.

Abbreviations: NC, negative control; SD, standard deviation.

CDH1 increased while that of vimentin reduced significantly (q-value was 18.67 and 5.99, respectively; $P<0.01$; Figure 6). The results suggested that miR-544a could downregulate $\mathrm{CDH} 1$ and upregulate vimentin in $95 \mathrm{C}$ cells.

\section{Discussion}

miRNA participates in tumor invasion and metastasis, ${ }^{6}$ but its mechanism is not very clear. In this study, we focused on two cell lines of NSCLC (95C and 95D), whose invasive ability is completely different. In the miRNA array assay, the miR-544a level was higher in 95D cells, which possess higher invasive ability. To avoid false positive results of the miRNA array, quantitative PCR was carried out to verify these results. Quantitative PCR also confirmed that the relative level of miR-544a in 95D was higher. In the transwell migration assay, the migration ability of $95 \mathrm{C}$ transfected with miR-544a mimic was higher, but that of 95D transfected with miR-544a inhibitor was lower. TargetScan is one of the most common software products used to predict an miRNA target gene. TargetScan showed that CDH1 may be the target gene of miR-544a. Luciferase assay also revealed that miR-544a could combine with CDH1 3'UTR and inhibit the expression of the reporter gene. In our previous work, we found that glycogen synthase kinase $3 \beta$ is another target of miR-544a and is regulated by $95 \mathrm{D}$ cells. ${ }^{7}$

It is accepted that downregulation of $\mathrm{CDH} 1$ and upregulation of vimentin can promote the invasion of lung cancer. In the present study, we found that in 95C transfected with
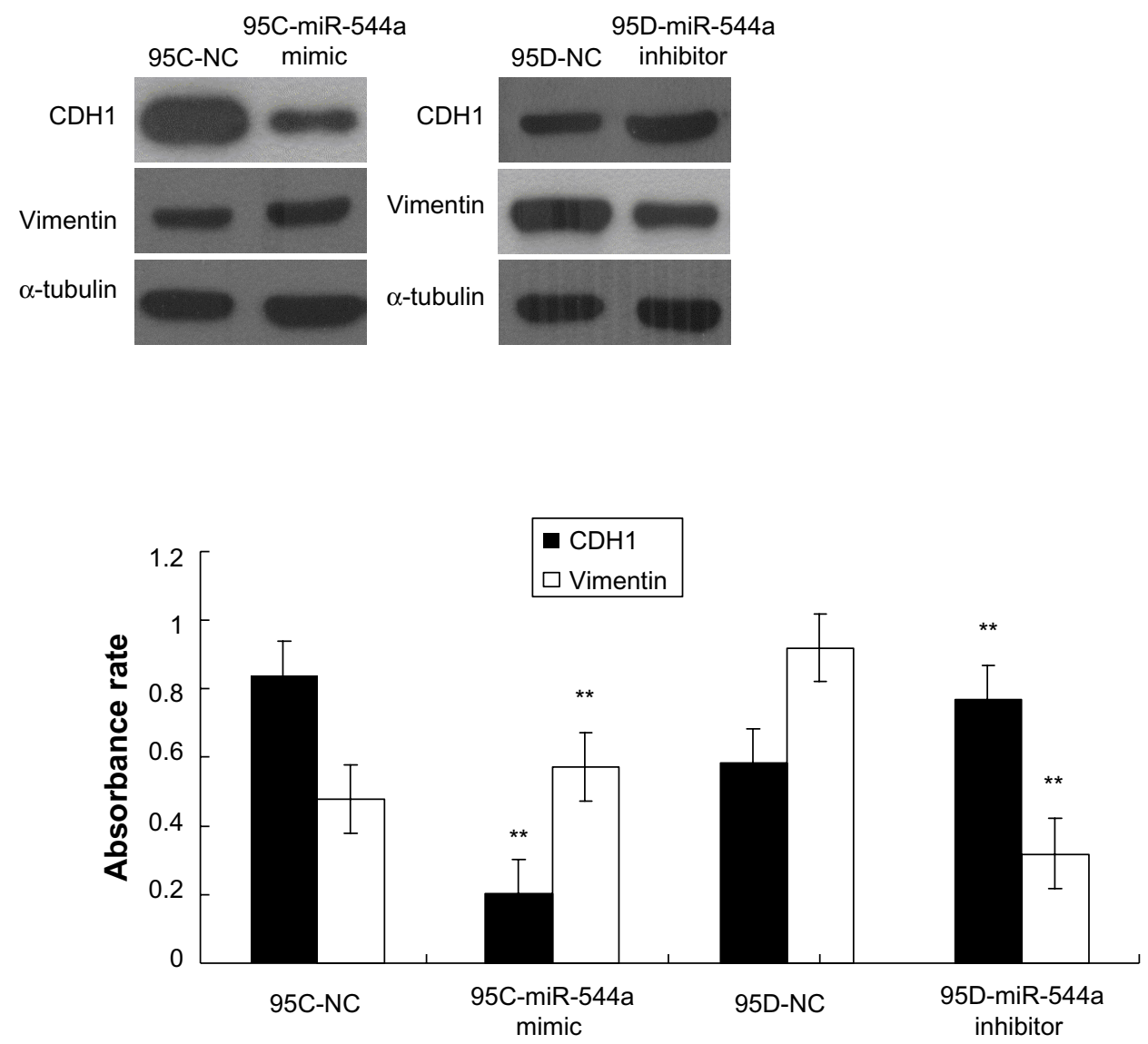

Figure $6 \mathrm{CDHI}$ (E-cadherin) and vimentin regulation by miR-544a in Western blot. In $95 \mathrm{C}$ transfected with miR-544a mimic, the level of CDHI (0.20I9 $\pm 0.0 \mathrm{I} 65)$ reduced and that of vimentin $(0.5735 \pm 0.0092)$ increased compared with $95 \mathrm{C}-\mathrm{NC}(* * P<0.0 \mathrm{I})$; while in $95 \mathrm{D}$ transfected with miR-544a inhibitor, the level of CDHI $(0.7694 \pm 0.014 \mathrm{I})$ increased and that of vimentin $(0.3200 \pm 0.0197)$ reduced significantly $(* * p<0.01)$.

Abbreviation: NC, negative control. 
miR-544a mimic, CDH1 levels reduced and vimentin levels increased. But in 95D transfected with miR-544a inhibitor, CDH1 levels increased and vimentin levels reduced. Downregulation of $\mathrm{CDH} 1$ is considered as the mark of epithelial-mesenchymal transition (EMT), which plays an important role in the process of tumor metastasis. ${ }^{8}$ EMT makes tumor's acquire invasive and metastatic ability. We found that in the process of EMT, CDH1 levels reduced and vimentin levels increased, which is consistent with the findings of Thiery and Lim. ${ }^{9}$ However, further in vivo studies are needed to confirm the role of miR-544a in invasion and metastasis of NSCLC and its regulation on CDH1 and vimentin.

In conclusion, our current study provided novel evidence that higher expression of miR-544a significantly suppresses $\mathrm{CDH} 1$, resulting in the promoting of the invasion and metastasis of NSCLC in vitro. The regulation of miR-544a on CDH1 provides novel insight into the metastasis of NSCLC, especially with respect to invasion and metastasis in vitro, and also represents a new potential therapeutic target for the treatment of NSCLC.

\section{Disclosure}

The authors report no conflicts of interest in this work.

\section{References}

1. Lam WK, Watkins DN. Lung cancer: future directions. Respirology. 2007;12(4):471-477.

2. Vimalraj S, Miranda PJ, Ramyakrishna B, et al. Regulation of breast cancer and bone metastasis by microRNAs. Dis Markers. 2013;35(5): 369-387.

3. Ma R, Zhang G, Wang H, et al. Downregulation of miR-544 in tissue, but not in serum, is a novel biomarker of malignant transformation in glioma. Oncol Lett. 2012;4(6):1321-1324.

4. Haga CL, Phinney DG. MicroRNAs in the imprinted DLK1-DIO3 region repress the epithelial-to-mesenchymal transition by targeting the TWIST1 protein signaling network. J Biol Chem. 2012;287(51): 42695-42707.

5. Zhi Q, Guo X, Guo L, et al. Oncogenic miR-544 is an important molecular target in gastric cancer. Anticancer Agents Med Chem. 2013;13(2):270-275.

6. Pencheva N, Tavazoie SF. Control of metastatic progression by microRNA regulatory networks. Nat Cell Biol. 2013;15(6): $546-554$.

7. Mo XM, Li HH, Li YT. Down regulation of GSK3 $\beta$ by miR-544a to maintain self-renewal ability of lung cancer stem cells. Oncol Lett. In press 2014.

8. Chen Y, Sun Y, Chen L, et al. miRNA-200c increases the sensitivity of breast cancer cells to doxorubicin through the suppression of E-cadherin-mediated PTEN/Akt signaling. Mol Med Rep. 2013;7(5): 1579-1584.

9. Thiery JP, Lim CT. Tumor dissemination: an EMT affair. Cancer Cell. 2013;23(3):272-273.
OncoTargets and Therapy

\section{Publish your work in this journal}

OncoTargets and Therapy is an international, peer-reviewed, open access journal focusing on the pathological basis of all cancers, potential targets for therapy and treatment protocols employed to improve the management of cancer patients. The journal also focuses on the impact of management programs and new therapeutic agents and protocols on

\section{Dovepress}

patient perspectives such as quality of life, adherence and satisfaction. The manuscript management system is completely online and includes a very quick and fair peer-review system, which is all easy to use. Visit http://www.dovepress.com/testimonials.php to read real quotes from published authors. 